\title{
On the Krasnoselskii-type fixed point theorems for the sum of continuous and asymptotically nonexpansive mappings in Banach spaces
}

\author{
Areerat Arunchai and Somyot Plubtieng*
}

\author{
* Correspondence: somyotp@ \\ nu.ac.th \\ Department of Mathematics, \\ Faculty of Science, Naresuan \\ University, Phitsanulok 65000, \\ Thailand
}

\begin{abstract}
In this article, we prove some results concerning the Krasnoselskii theorem on fixed points for the sum $A+B$ of a weakly-strongly continuous mapping and an asymptotically nonexpansive mapping in Banach spaces. Our results encompass a number of previously known generalizations of the theorem.
\end{abstract}

Keywords: Krasnoselskii's fixed point theorem, asymptotically nonexpansive mapping, weakly-strongly continuous mapping, uniformly asymptotically regular, measure of weak noncompactness

\section{Introduction}

As is well known, Krasnoselskii's fixed point theorem has a wide range of applications to nonlinear integral equations of mixed type (see [1]). It has also been extensively employed to address differential and functional differential equations. His theorem actually combines both the Banach contraction principle and the Schauder fixed point theorem, and is useful in establishing existence theorems for perturbed operator equations. Since then, there have appeared a large number of articles contributing generalizations or modifications of the Krasnoselskii fixed point theorem and their applications (see [2]-[21]).

The study of asymptotically nonexpansive mappings concerning the existence of fixed points have become attractive to the authors working in nonlinear analysis. Goebel and Kirk [22] introduced the concept of asymptotically nonexpansive mappings in Banach spaces and proved a theorem on the existence of fixed points for such mappings in uniformly convex Banach spaces. In 1971, Cain and Nashed [23] generalized to locally convex spaces a well known fixed point theorem of Krasnoselskii for a sum of contraction and compact mappings in Banach spaces. The class of asymptotically nonexpansive mappings includes properly the class of nonexpansive mappings as well as the class of contraction mappings. Recently, Vijayaraju [21] proved by using the same method some results concerning the existence of fixed points for a sum of nonexpansive and continuous mappings and also a sum of asymptotically nonexpansive and continuous mappings in locally convex spaces. Very recently, Agarwal et al. [1] proved some existence theorems of a fixed point for the sum of a weakly-strongly

\section{SpringerOpen ${ }^{\circ}$}

(C) 2011 Arunchai and Plubtieng; licensee Springer. This is an Open Access article distributed under the terms of the Creative Commons Attribution License (http://creativecommons.org/licenses/by/2.0), which permits unrestricted use, distribution, and reproduction in any medium, provided the original work is properly cited. 
continuous mapping and a nonexpansive mapping on a Banach space and under Krasnoselskii-, Leray Schauder-, and Furi-Pera-type conditions.

Motivated and inspired by Agarwal et al. [1] and Vijayaraju [21], in this article we will prove some new generalized forms of the Krasnoselskii theorem on fixed points for the sum $A+B$ of a weakly-strongly continuous mapping and an asymptotically nonexpansive mapping in Banach spaces. These results encompass a number of previously known generalizations of the theorem.

\section{Preliminaries}

Let $M$ be a nonempty subset of a Banach space $X$ and $T: M \rightarrow X$ be a mapping. We say that $T$ is weakly-strongly continuous if for each sequence $\left\{x_{n}\right\}$ in $M$ which converges weakly to $x$ in $M$, the sequence $\left\{T x_{n}\right\}$ converges strongly to $T x$. The mapping $T$ is nonexpansive if $\|T x-T y\| \leq|| x-y||$ for all $x, y \in M$, and $T$ is asymptotically nonexpansive (see [22]) if there exists a sequence $\left\{k_{n}\right\}$ with $k_{n} \geq 1$ for all $n$ and $\lim _{n \rightarrow \infty} k_{n}=1$ such that $\left\|T^{n} x-T^{n} y\right\| \leq k_{n}\|x-y\|$ for all $n \geq 1$ and $x, y \in M$.

Definition 2.1. [21] If $B$ and $A$ map $M$ into $X$, then $B$ is called a uniformly asymptotically regular with respect to $A$ if, for each $\varepsilon>0$ there exists $n_{0} \in \mathbb{N}$, such that

$$
\left\|B^{n}(x)-B^{n-1}(x)+A(x)\right\| \leq \varepsilon
$$

for all $n \geq n_{0}$ and all $x \in M$.

Now, let us recall some definitions and results which will be needed in our further considerations. Let $X$ be a Banach space, $\Omega(X)$ is the collection of all nonempty bounded subsets of $X$, and $\mathcal{W}(X)$ is the subset of $\Omega(X)$ consisting of all weak compact subsets of $X$. Let $B_{r}$ denote the closed ball in $X$ countered at 0 with radius $r>0$. In [24], De Blasi introduced the following mapping $\omega: \Omega(X) \rightarrow[0, \infty)$ defined by

$$
\omega(M)=\inf \left\{r>0: \text { there exists a set } N \in \mathcal{W}(X) \text { such that } M \subseteq N+B_{r}\right\},
$$

for all $M \in \Omega(X)$. For completeness, we recall some properties of $\omega(\cdot)$ needed below (for the proofs we refer the reader to [24]).

Lemma 2.2. [24] Let $M_{1}$ and $M_{2} \in \Omega(X)$, then we have

(i) If $M_{1} \subseteq M_{2}$, then $\omega\left(M_{1}\right) \leq \omega\left(M_{2}\right)$.

(ii) $\omega\left(M_{1}\right)=0$ if and only if $M_{1}$ is relatively weakly compact.

(iii) $\omega\left(\overline{M_{1}^{w}}\right)=\omega\left(M_{1}\right)$, where $\overline{M_{1}^{w}}$ is the weak closure of $M_{1}$.

(iv) $\omega\left(\lambda M_{1}\right)=|\lambda| \omega\left(M_{1}\right)$ for all $\lambda \in \mathbb{R}$.

(v) $\omega\left(\operatorname{co}\left(M_{1}\right)\right)=w\left(M_{1}\right)$.

(vi) $\omega\left(M_{1}+M_{2}\right) \leq \omega\left(M_{1}\right)+\omega\left(M_{2}\right)$.

(vii) If $\left(M_{n}\right)_{n \geq 1}$ is a decreasing sequence of nonempty, bounded and weakly closed subsets of $X$ with $\lim _{n \rightarrow \infty} \omega\left(M_{n}\right)=0$, then $\bigcap_{n=1}^{\infty} M_{n} \neq$ Øand $\omega\left(\bigcap_{n=1}^{\infty} M_{n}\right)=0$, i.e., $\bigcap_{n=1}^{\infty} M_{n}$ is relatively weakly compact.

Throughout this article, a measure of weak noncompactness will be a mapping $\psi$ : $\Omega(X) \rightarrow[0, \infty)$ which satisfies the assumptions (i)-(vii) cited in Lemma 2.2.

Definition 2.3. [25] Let $M$ be a closed subset of $X$ and $I, T: M \rightarrow M$ be two mappings. A mapping $T$ is said to be demiclosed at the zero, if for each sequence $\left\{x_{n}\right\}$ in $M$, the conditions $x_{n} \rightarrow x_{0}$ weakly and $T x_{n} \rightarrow 0$ strongly imply $T x_{0}=0$. 
Lemma 2.4. [26]-[29] Let $X$ be a uniformly convex Banach space, $M$ be a nonempty closed convex subset of $X$, and let $T: M \rightarrow M$ be an asymptotically nonexpansive mapping with $F(T) \neq \varnothing$. Then $I-T$ is demiclosed at zero, i.e., for each sequence $\left\{x_{n}\right\}$ in $M$, if $\left\{x_{n}\right\}$ converges weakly to $q \in M$ and $\left\{(I-T) x_{n}\right\}$ converges strongly to 0 , then $(I-T) q=0$.

Definition 2.5. $[1,13]$ Let $X$ be a Banach space and let $\psi$ be a measure of weak noncompactness on $X$. A mapping $B: D(B) \subseteq X \rightarrow X$ is said to be $\psi$-contractive if it maps bounded sets into bounded sets and there is a $\beta \in[0,1)$ such that $\psi(B(S)) \leq \beta \psi(S)$ for all bounded sets $S \subseteq D(B)$. The mapping $B: D(B) \subseteq X \rightarrow X$ is said to be $\psi$-condensing if it maps bounded sets into bounded sets and $\psi(B(S))<\psi(S)$ whenever $S$ is a bounded subset of $D(B)$ such that $\psi(S)>0$.

Let $\mathcal{J}$ be a nonlinear operator from $D(\mathcal{J}) \subseteq X$ into $X$. In the next section, we will use the following two conditions:

$(\mathcal{H} 1)$ If $\left(x_{n}\right)_{n \in \mathbb{N}}$ is a weakly convergent sequence in $D(\mathcal{J})$, then $\left(\mathcal{J} x_{n}\right)_{n \in \mathbb{N}}$ has a strongly convergent subsequence in $X$.

$(\mathcal{H} 2)$ If $\left(x_{n}\right)_{n \in \mathbb{N}}$ is a weakly convergent sequence in $D(\mathcal{J})$, then $\left(\mathcal{J} x_{n}\right)_{n \in \mathbb{N}}$ has a weakly convergent subsequence in $X$.

Remark 2.6. 1. Operators satisfying $(\mathcal{H} 1)$ or $(\mathcal{H} 2)$ are not necessarily weakly continuous (see $[12,19,30])$.

2. Every $w$-contractive mapping satisfies $(\mathcal{H} 2)$.

3. A mapping $\mathcal{J}$ satisfies $(\mathcal{H} 2)$ if and only if it maps relatively weakly compact sets into relatively weakly compact ones (use the Eberlein-Šmulian theorem [31]).

4. A mapping $\mathcal{J}$ satisfies $(\mathcal{H} 1)$ if and only if it maps relatively weakly compact sets into relatively compact ones.

5. The condition $(\mathcal{H} 2)$ holds true for every bounded linear operator.

The following fixed point theorems are crucial for our purposes.

Lemma 2.7. [12] Let $M$ be a nonempty closed bounded convex subset of a Banach space $X$. Suppose that $A: M \rightarrow X$ and $B: X \rightarrow X$ satisfying:

(i) A is continuous, AM is relatively weakly compact and A satisfies $(\mathcal{H} 1)$

(ii) $B$ is a strict contraction satisfying $(\mathcal{H} 2)$,

(iii) $A x+B y \in M$ for all $x, y \in M$.

Then, there is an $x \in M$ such that $A x+B x=x$.

Lemma 2.8. [20] Let $M$ be a nonempty closed bounded convex subset of a Banach space $X$. Suppose that $A: M \rightarrow X$ and $B: M \rightarrow X$ are sequentially weakly continuous such that:

(i) AM is relatively weakly compact,

(ii) $B$ is a strict contraction,

(iii) $A x+B y \in M$ for all $x, y \in M$.

Then, there is an $x \in M$ such that $A x+B x=x$.

Lemma 2.9. [1] Let $X$ be a Banach space and let $\psi$ be measure of weak noncompactness on $X$. Let $Q$ and $C$ be closed, bounded, convex subset of $X$ with $Q \subseteq C$. In addition, 
let $U$ be a weakly open subset of $Q$ with $0 \in U$, and $F: \overline{U^{w}} \rightarrow C$ a weakly sequentially continuous and $\psi$-condensing mapping. Then either

F has a fixed point,

or

thereis a point $u \in \partial_{\mathrm{Q}} U$ and, $\lambda \in(0,1)$ with $u=\lambda F u$

here $\partial_{Q} U$ is the weak boundary of $U$ in $Q$.

Lemma 2.10. [1] Let $X$ be a Banach space and $B: X \rightarrow X$ a k-Lipschitzian mapping, that is

$$
\forall x, y \in X,\|B x-B y\| \leq k\|x-y\| .
$$

In addition, suppose that $B$ verifies $(\mathcal{H} 2)$. Then for each bounded subset $S$ of $X$, we have $\psi(B S) \leq k \psi(S)$;

here, $\psi$ is the De Blasi measure of weak noncompactness.

Lemma 2.11. [15,32] Let $X$ be a Banach space with $C \subseteq X$ closed and convex. Assume $U$ is a relatively open subset of $C$ with $0 \in U, F(\bar{U})$ bounded and $F: \bar{U} \rightarrow C$ a condensing mapping. Then, either $F$ has a fixed point in $\bar{U}$ or there is a point $u \in \partial U$ and $\lambda \in$ $(0,1)$ with $u=\lambda F(u)$; here $\bar{U}$ and $\partial U$ denote the closure of $U$ in $C$ and the boundary of $U$ in $C$, respectively.

Lemma 2.12. $[15,32]$ Let $X$ be a Banach space and $Q$ a closed convex bounded subset of $X$ with $0 \in Q$. In addition, assume $F: Q \rightarrow X$ a condensing mapping with if $\left\{\left(x_{j}, \lambda_{j}\right)\right\}_{j=1}^{+\infty}$ is a sequence in $\partial Q \times[0,1]$ converging to $(x, \lambda)$ with $X=\lambda F(x)$ and $0<\lambda$ $<1$, then $\lambda_{j} F\left(x_{j}\right) \in Q$ for $j$ sufficiently large, holding. Then $F$ has a fixed point.

\section{Main results}

Now, we are ready to state and prove the main result of this section.

Theorem 3.1. Let $M$ be a nonempty bounded closed convex subset of a Banach space $X$. Let $A: M \rightarrow X$ and $B: M \rightarrow M$ satisfy the following:

(i) A is weakly-strongly continuous, and AM is relatively weakly compact,

(ii) $B$ is an asymptotically nonexpansive mapping with a sequence $\left(k_{n}\right) \subset[1, \infty)$ satisfying $(\mathcal{H} 2)$,

(iii) if $\left(x_{n}\right)$ is a sequence of $M$ such that $\left((I-B) x_{n}\right)$ is weakly convergent, then the sequence $\left(x_{n}\right)$ has a weakly convergent subsequence,

(iv) $I-B$ is demiclosed,

(v) $B^{n} x+A y \in M$ for all $x, y \in M$ and $n=1,2, \ldots$,

(vi) $B$ is uniformly asymptotically regular with respect to $A$.

Then, there is an $x \in M$ such that $A x+B x=x$.

Proof. Suppose first that $0 \in M$ and let $a_{n}:=\left(1-\frac{1}{n}\right) / k_{n}$ for all $n \in \mathbb{N}$. By hypothesis (v), we have

$$
a_{n} B^{n} x+a_{n} A y \in M \text { for all } n \in \mathbb{N} \text { and } x, y \in M .
$$


Since $B$ is asymptotically nonexpansive, it follows that

$$
\begin{aligned}
\left\|a_{n} B^{n} x-a_{n} B^{n} y\right\| & =a_{n}\left\|B^{n} x-B^{n} y\right\| \\
& \leq a_{n} k_{n}\|x-y\| \\
& =\left(1-\frac{1}{n}\right)\|x-y\| \text { forall } x, y \in M .
\end{aligned}
$$

Hence, $a_{n} B^{n}$ is contraction on $M$. Therefore, by Lemma 2.7 , there is an $x_{n} \in M$ such that

$$
a_{n}\left(B^{n} x_{n}+A x_{n}\right)=x_{n}
$$

for all $n \in \mathbb{N}$. This implies that

$$
x_{n}-\left(B^{n} x_{n}+A x_{n}\right)=\left(a_{n}-1\right)\left(B^{n} x_{n}+A x_{n}\right) \rightarrow 0 \text { as } n \rightarrow \infty
$$

since $a_{n} \rightarrow 1$ as $n \rightarrow \infty$ and $M$ is bounded and $B^{n} x+A y \in M$ for all $x, y \in M$. Since $B$ is uniformly asymptotically regular with respect to $A$, it follows that

$$
B^{n} x_{n}-B^{n-1} x_{n}+A x_{n} \rightarrow 0 \text { as } n \rightarrow \infty \text {. }
$$

From (3.3) and (3.4), we obtain

$$
x_{n}-B^{n-1} x_{n} \rightarrow 0 \text { as } n \rightarrow \infty .
$$

Now, it is noted that

$$
\begin{aligned}
\left\|x_{n}-B x_{n}-A x_{n}\right\| & =\left\|x_{n}-(B+A) x_{n}\right\| \\
& \leq\left\|x_{n}-\left(B^{n}+A\right) x_{n}\right\|+\left\|\left(B^{n}+A\right) x_{n}-(B+A) x_{n}\right\| \\
& =\left\|x_{n}-\left(B^{n}+A\right) x_{n}\right\|+\left\|B^{n} x_{n}-B x_{n}\right\| \\
& \leq\left\|x_{n}-\left(B^{n}+A\right) x_{n}\right\|+k_{1}\left\|B^{n-1} x_{n}-x_{n}\right\| .
\end{aligned}
$$

Using (3.3) and (3.5) in (3.6), we get

$$
x_{n}-B x_{n}-A x_{n} \rightarrow 0 \text { as } n \rightarrow \infty \text {. }
$$

Using the fact that $A M$ is weakly compact and passing eventually to a subsequence, we may assume that $\left\{A x_{n}\right\}$ converges weakly to some $y \in M$. By (3.7), we have

$$
(I-B) x_{n} \rightarrow y \text {. }
$$

By hypothesis (iii), the sequence $\left\{x_{n}\right\}$ has a subsequence $\left\{x_{n_{k}}\right\}$ which converges weakly to some $x \in M$. Since $A$ is weakly-strongly continuous, $\left\{A x_{n_{k}}\right\}$ converges strongly to $A x$.

Hence, we observe that

$$
x_{n_{k}}-B x_{n_{k}}=(I-B) x_{n_{k}} \rightarrow A x \text { as } k \rightarrow \infty .
$$

Hence, by the demiclosedness of $I-B$, we have $A x+B x=x$.

To complete the proof, it remains to consider the case $0 \notin M$. In such a case, let us fix any element $x_{0} \in M$ and let $M_{0}=\left\{x-x_{0}, x \in M\right\}$. Define two mappings $A_{0}: M_{0}$ $\rightarrow X$ and $B_{0}: M_{0} \rightarrow M$ by $A_{0}\left(x-x_{0}\right)=A x-\frac{1}{2} x_{0}$ and $B_{0}\left(x-x_{0}\right)=B x-\frac{1}{2} x_{0}$, for $x \in$ $M$. By the result of the first case for $A_{0}$ and $B_{0}$, we have an $x \in M$ such that $A_{0}\left(x-x_{0}\right)$ $+B_{0}\left(x-x_{0}\right)=x-x_{0}$. Hence $A x+B x=x$.

Corollary 3.2. Let $M$ be a nonempty bounded closed convex subset of a uniformly convex Banach space $X$. Let $A: M \rightarrow X$ and $B: M \rightarrow M$ satisfy the following: 
(i) A is weakly-strongly continuous,

(ii) $B$ is an asymptotically nonexpansive mapping with a sequence $\left(k_{n}\right) \subset[1, \infty)$,

(iii) $B^{n} x+A y \in M$ for all $x, y \in M$, and $n=1,2, \ldots$,

(iv) $B$ is uniformly asymptotically regular with respect to $A$.

Then, there is an $x \in M$ such that $A x+B x=x$.

Our next result is the following:

Theorem 3.3. Let $M$ be a nonempty bounded closed convex subset of a Banach space $X$. Suppose that $A: M \rightarrow X$ and $B: M \rightarrow M$ are two weakly sequentially continuous mappings that satisfy the following:

(i) AM is relatively weakly compact,

(ii) $B$ is an asymptotically nonexpansive mapping with a sequence $\left(k_{n}\right) \subset[1, \infty)$,

(iii) if $\left(x_{n}\right)$ is a sequence of $M$ such that $\left((I-B) x_{n}\right)$ is weakly convergent, then the sequence $\left(x_{n}\right)$ has a weakly convergent subsequence,

(iv) $B^{n} x+A y \in M$ for all $x, y \in M$, and $n=1,2, \ldots$,

(v) $B$ is uniformly asymptotically regular with respect to $A$.

Then, there is an $x \in M$ such that $A x+B x=x$.

Proof. Without loss of generality, we may assume that $0 \in M$. Let $a_{n}:=\left(1-\frac{1}{n}\right) / k_{n} \in(0,1)$ for all $n \in \mathbb{N}$. By hypothesis $(i v)$, we have

$$
a_{n} B^{n} x+a_{n} A y \in M \text { for all } n \in \mathbb{N} \text { and } x, y \in M \text {. }
$$

Since $B$ is asymptotically nonexpansive, it follows that

$$
\begin{aligned}
\left\|a_{n} B^{n} x-a_{n} B^{n} y\right\| & =a_{n}\left\|B^{n} x-B^{n} y\right\| \\
& \leq a_{n} k_{n}\|x-y\| \\
& =\left(1-\frac{1}{n}\right)\|x-y\|, \text { for all } x, y \in M .
\end{aligned}
$$

Hence, $a_{n} B^{n}$ is a contraction on $M$. By Lemma 2.8 , there is a $x_{n} \in M$ such that

$$
a_{n}\left(B^{n} x_{n}+A x_{n}\right)=x_{n}
$$

for all $n \in \mathbb{N}$. This implies that

$$
x_{n}-\left(B^{n} x_{n}+A x_{n}\right)=\left(a_{n}-1\right)\left(B^{n} x_{n}+A x_{n}\right) \rightarrow 0 \text { as } n \rightarrow \infty .
$$

Since $B$ is uniformly asymptotically regular with respect to $A$, it follows that

$$
B^{n} x_{n}-B^{n-1} x_{n}+A x_{n} \rightarrow 0 \text { as } n \rightarrow \infty .
$$

From (3.12) and (3.13), we obtain

$$
x_{n}-B^{n-1} x_{n} \rightarrow 0 \text { as } n \rightarrow \infty .
$$

Now, it is noted that

$$
\begin{aligned}
\left\|x_{n}-B x_{n}-A x_{n}\right\| & =\left\|x_{n}-(B+A) x_{n}\right\| \\
& \leq\left\|x_{n}-\left(B^{n}+A\right) x_{n}\right\|+\left\|\left(B^{n}+A\right) x_{n}-(B+A) x_{n}\right\| \\
& =\left\|x_{n}-\left(B^{n}+A\right) x_{n}\right\|+\left\|B^{n} x_{n}-B x_{n}\right\| \\
& \leq\left\|x_{n}-\left(B^{n}+A\right) x_{n}\right\|+k_{1}\left\|B^{n-1} x_{n}-x_{n}\right\| .
\end{aligned}
$$


Using (3.12) and (3.14) in (3.15), we get

$$
x_{n}-B x_{n}-A x_{n} \rightarrow 0 \text { as } n \rightarrow \infty .
$$

Using the fact that $A M$ is weakly compact and passing eventually to a subsequence, we may assume that $\left\{A x_{n}\right\}$ converges weakly to some $y \in M$. Hence, by (3.16)

$$
(I-B) x_{n} \rightarrow \gamma .
$$

By hypothesis (iii), the sequence $\left\{x_{n}\right\}$ has a subsequence $\left\{x_{n_{k}}\right\}$ which converges weakly to some $x \in M$. Since $A$ and $B$ are weakly sequentially continuous, $\left\{A x_{n_{k}}\right\}$ converges weakly to $A x$, and $\left\{B x_{n_{k}}\right\}$ converges weakly to $B x$. Hence, $A x+B x=x$. $\square$

Theorem 3.4. Let $Q$ and $C$ be closed bounded convex subset of a Banach space $X$ with $Q \subseteq C$. In addition, let $U$ be a weakly open subset of $Q$ with $0 \in U$, $A: \overline{U^{w}} \rightarrow X$ and $B: X \rightarrow X$ are two weakly sequentially continuous mappings satisfying the following:

(i) $A\left(\overline{U^{w}}\right)$ is a relatively weakly compact,

(ii) $B$ is an asymptotically nonexpansive mapping with a sequence $\left(k_{n}\right) \subset[1, \infty)$,

(iii) if $\left(x_{n}\right)$ is a sequence of $M$ such that $\left((I-B) x_{n}\right)$ is weakly convergent, then the sequence $\left(x_{n}\right)$ has a weakly convergent subsequence,

(iv) $B^{n} x+A y \in C$ for all $x, y \in \overline{U^{w}}$, and $n=1,2, \ldots$,

(v) $B$ is uniformly asymptotically regular with respect to $A$.

Then, either

$$
A+B \text { has a fixed point, }
$$

or

there is a point $u \in \partial_{Q} U$ and $\lambda \in(0,1)$ with $u=\lambda\left(A+B^{n}\right) u$

here, $\partial_{Q} U$ is the weak boundary of $U$ in $Q$.

Proof. Let $a_{n}:=\left(1-\frac{1}{n}\right) / k_{n} \in(0,1)$ for all $n \in \mathbb{N}$. We first show that the mapping $F_{n}$ $=a_{n} A+a_{n} B^{n}$ is $\psi$-contractive with constant $a_{n}$. To see that, let $S$ be a bounded subset of $\overline{U^{w}}$. Using the homogeneity and the subadditivity of the De Blasi measure of weak noncompactness, we obtain

$$
\psi\left(F_{n}(S)\right) \leq \psi\left(a_{n} A S+a_{n} B^{n} S\right) \leq a_{n} \psi(A S)+a_{n} \psi\left(B^{n} S\right) .
$$

Keeping in mind that $A$ is weakly compact and using Lemma 2.10, we deduce that

$$
\psi\left(F_{n}(S)\right) \leq a_{n} k_{n} \psi(S) .
$$

This proves that $F_{n}$ is $\psi$-contractive with constant $a_{n}$. Moreover, taking into account that $0 \in U$ and using assumption (iv), we infer that $F_{n}$ map $\overline{U^{w}}$ into C. Next, we suppose that (3.19) does not occur, and $F_{n}$ does not have a fixed point on $\partial_{Q} U$ (otherwise we are finished since (3.18) occurs). If there exists a $u \in \partial_{Q} U$, and $\lambda \in(0,1)$ with $u=$ $\lambda F_{n} u$ then $u=\lambda a_{n} A u+\lambda a_{n} B^{n} u$. It is impossible since $\lambda a_{n} \in(0,1)$. By Lemma 2.9, there exists $x_{n} \in \overline{U^{w}}$ such that

$$
x_{n}=F_{n} x_{n}=a_{n} A x_{n}+a_{n} B^{n} x_{n}
$$


for all $n \in \mathbb{N}$. This implies that

$$
x_{n}-\left(B^{n} x_{n}+A x_{n}\right)=\left(a_{n}-1\right)\left(B^{n} x_{n}+A x_{n}\right) \rightarrow 0 \text { as } n \rightarrow \infty .
$$

Since $B$ is uniformly asymptotically regular with respect to $A$, it follows that

$$
B^{n} x_{n}-B^{n-1} x_{n}+A x_{n} \rightarrow 0 \text { as } n \rightarrow \infty \text {. }
$$

From (3.20) and (3.21), we obtain

$$
x_{n}-B^{n-1} x_{n} \rightarrow 0 \text { as } n \rightarrow \infty .
$$

Now, it is noted that

$$
\begin{aligned}
\left\|x_{n}-B x_{n}-A x_{n}\right\| & =\left\|x_{n}-(B+A) x_{n}\right\| \\
& \leq\left\|x_{n}-\left(B^{n}+A\right) x_{n}\right\|+\left\|\left(B^{n}+A\right) x_{n}-(B+A) x_{n}\right\| \\
& =\left\|x_{n}-\left(B^{n}+A\right) x_{n}\right\|+\left\|B^{n} x_{n}-B x_{n}\right\| \\
& \leq\left\|x_{n}-\left(B^{n}+A\right) x_{n}\right\|+k_{1}\left\|B^{n-1} x_{n}-x_{n}\right\| .
\end{aligned}
$$

Using (3.20) and (3.22) in (3.23), we get

$$
x_{n}-B x_{n}-A x_{n} \rightarrow 0 \text { as } n \rightarrow \infty \text {. }
$$

Since $A M$ is weakly compact and passing eventually to a subsequence, we may assume that $\left\{A x_{n}\right\}$ converges weakly to some $y \in \bar{U}$. Thus, we have

$$
(I-B) x_{n} \rightarrow y \text {. }
$$

By hypothesis (iii), the sequence $\left\{x_{n}\right\}$ has a subsequence $\left\{x_{n_{k}}\right\}$ which converges weakly to some $x \in \bar{U}$. Since $A$ and $B$ are weakly sequentially continuous, $\left\{A x_{n_{k}}\right\}$ converges weakly to $A x$, and $\left\{B x_{n_{k}}\right\}$ converges weakly to $B x$. Hence, $A x+B x=x$.

Theorem 3.5. Let $U$ be a bounded open convex set in a Banach space $X$ with $0 \in U$. Suppose $A: \bar{U} \rightarrow$ Xand $B: X \rightarrow X$ are continuous mappings satisfying the following:

(i) $A(\bar{U})$ is compact, and $A$ is weakly-strongly continuous,

(ii) $B$ is an asymptotically nonexpansive mapping with a sequence $\left(k_{n}\right) \subset[1, \infty)$, and $I$ - B is demiclosed,

(iii) if $\left(x_{n}\right)$ is a sequence of $\bar{U}$ such that $\left((I-B) x_{n}\right)$ is weakly convergent, then the sequence $\left(x_{n}\right)$ has a weakly convergent subsequence,

(iv) $B$ is uniformly asymptotically regular with respect to $A$.

Then, either

$$
A+B \text { has a fixed point, }
$$

or

there is a point $u \in \partial U$ and $\lambda \in(0,1)$ with $u=\lambda B^{n} u+\lambda A u$.

Proof. Suppose (3.27) does not occur and let $a_{n}:=\left(1-\frac{1}{n}\right) / k_{n} \in(0,1)$ for all $n \in \mathbb{N}$. The mapping $F_{n}:=a_{n} A+a_{n} B^{n}$ is the sum of a compact and a strict contraction. This implies that $F_{n}$ is a condensing mapping (see [13]). By Lemma 2.11, we deduce that there is an $x_{n} \in \bar{U}$ such that

$$
x_{n}=F_{n} x_{n}=a_{n} A x_{n}+a_{n} B^{n} x_{n}
$$


for all $n \in \mathbb{N}$. This implies that

$$
x_{n}-\left(B^{n} x_{n}+A x_{n}\right)=\left(a_{n}-1\right)\left(B^{n} x_{n}+A x_{n}\right) \rightarrow 0 \text { as } n \rightarrow \infty .
$$

Since $B$ is uniformly asymptotically regular with respect to $A$, it follows that

$$
B^{n} x_{n}-B^{n-1} x_{n}+A x_{n} \rightarrow 0 \text { as } n \rightarrow \infty \text {. }
$$

From (3.28) and (3.29), we obtain

$$
x_{n}-B^{n-1} x_{n} \rightarrow 0 \text { as } n \rightarrow \infty .
$$

Now, it is noted that

$$
\begin{aligned}
\left\|x_{n}-B x_{n}-A x_{n}\right\| & =\left\|x_{n}-(B+A) x_{n}\right\| \\
& \leq\left\|x_{n}-\left(B^{n}+A\right) x_{n}\right\|+\left\|\left(B^{n}+A\right) x_{n}-(B+A) x_{n}\right\| \\
& =\left\|x_{n}-\left(B^{n}+A\right) x_{n}\right\|+\left\|B^{n} x_{n}-B x_{n}\right\| \\
& \leq\left\|x_{n}-\left(B^{n}+A\right) x_{n}\right\|+k_{1}\left\|B^{n-1} x_{n}-x_{n}\right\| .
\end{aligned}
$$

Using (3.28) and (3.30) in (3.31), we get

$$
x_{n}-B x_{n}-A x_{n} \rightarrow 0 \text { as } n \rightarrow \infty \text {. }
$$

Since $A M$ is weakly compact and passing eventually to a subsequence, we may assume that $\left\{A x_{n}\right\}$ converges weakly to some $y \in \bar{U}$. This implies that

$$
(I-B) x_{n} \rightarrow y \text {. }
$$

By hypothesis (iii), the sequence $\left\{x_{n}\right\}$ has a subsequence $\left\{x_{n_{k}}\right\}$ which converges weakly to some $x \in \bar{U}$. Since $A$ is weakly-strongly continuous, $\left\{A x_{n_{k}}\right\}$ converges strongly to $A x$.

Consequently

$$
x_{n_{k}}-B x_{n_{k}}=(I-B) x_{n_{k}} \rightarrow A x \text { as } k \rightarrow \infty .
$$

By the demiclosedness of $I-B$, we have $A x+B x=x$. $\square$

Corollary 3.6. Let $U$ be a bounded open convex set in a uniformly convex Banach space $X$ with $0 \in U$. Suppose $A: \bar{U} \rightarrow X$ and $B: X \rightarrow X$ are continuous mappings satisfying the following.

(i) $A(\bar{U})$ is compact, and $A$ is weakly-strongly continuous,

(ii) $B$ is an asymptotically nonexpansive mapping with a sequence $\left(k_{n}\right) \subset[1, \infty)$,

(iii) $B$ is uniformly asymptotically regular with respect to $A$.

Then, either

$$
A+B \text { has a fixed point, }
$$

or

there is a point $u \in \partial U$ and $\lambda \in(0,1)$ with $u=\lambda B^{n} u+\lambda A u$.

Theorem 3.7. Let $Q$ be a closed convex bounded set in a Banach space $X$ with $0 \in$ $Q$. Suppose $A: Q \rightarrow X$ and $B: X \rightarrow X$ are continuous mappings satisfying the following:

(i) $A(Q)$ is compact, and $A$ is weakly-strongly continuous, 
(ii) $B$ is an asymptotically nonexpansive mapping with a sequence $\left(k_{n}\right) \subset[1, \infty)$, and $I-B$ is demiclosed,

(iii) if $\left(x_{n}\right)$ is a sequence of $\bar{U}$ such that $\left((I-B) x_{n}\right)$ is weakly convergent, then the sequence $\left(x_{n}\right)$ has a weakly convergent subsequence,

(iv) if $\left\{\left(x_{j}, \lambda_{j}\right)\right\}_{j=1}^{+\infty}$ is a sequence of $\partial Q \times[0,1]$ converging to $(x, \lambda)$ with $X=\lambda A x+$ $\lambda B^{n} x$ and $0 \leq \lambda<1$, then $\lambda_{j} A x_{j}+\lambda_{j} B^{n} x_{j} \in Q$ for $j$ sufficiently large,

(v) $B$ is uniformly asymptotically regular with respect to $A$.

Then, $A+B$ has a fixed point in $Q$.

Proof. We first define $F_{n}:=a_{n} A+a_{n} B^{n}$, where $a_{n}:=\left(1-\frac{1}{n}\right) / k_{n} \in(0,1)$ for all $n \in \mathbb{N}$. Since $F_{n}$ is the sum of a compact mapping and a strict contraction mapping, it follows that $F_{n}$ is a condensing mapping. For any let fixed $n$, we have $\left\{\left(\gamma_{j}, \lambda_{j}\right)\right\}_{j=1}^{+\infty}$ is a sequence of $\partial Q \times[0,1]$ converging to $(y, \lambda)$ with $y=\lambda F_{n}(y)$ and $0 \leq \lambda<1$. Then $y=a_{n} \lambda A y+$ $a_{n} \lambda B^{n} y$. From assumption (iv), it follows that $a_{n} \lambda_{j} A y_{j}+a_{n} \lambda_{j} B^{n} y_{j} \in Q$ for $j$ sufficiently large. Applying Lemma 2.12 to $F_{n}$, we deduce that there is an $x_{n} \in Q$ such that

$$
x_{n}=F_{n} x_{n}=a_{n} A x_{n}+a_{n} B^{n} x_{n} .
$$

As in Theorem 3.5 this implies that

$$
(I-B) x_{n} \rightarrow y \text {. }
$$

By hypothesis $(i i i)$, the sequence $\left\{x_{n}\right\}$ has a subsequence $\left\{x_{n_{k}}\right\}$ which converges weakly to some $x \in Q$. Since $A$ is weakly-strongly continuous, $\left\{A x_{n_{k}}\right\}$ converges strongly to $A x$.

It follows that

$$
x_{n_{k}}-B x_{n_{k}}=(I-B) x_{n_{k}} \rightarrow A x \text { as } k \rightarrow \infty .
$$

Hence, by the demiclosedness of $I-B$, we have $A x+B x=x$.

Corollary 3.8. Let $Q$ be a closed convex bounded set in a uniformly convex Banach space $X$ with $0 \in Q$. Suppose $A: Q \rightarrow X$ and $B: X \rightarrow X$ are continuous mappings satisfying the following:

(i) $A(Q)$ is compact and $A$ is weakly-strongly continuous,

(ii) $B$ is an asymptotically nonexpansive mapping with a sequence $\left(k_{n}\right) \subset[1, \infty)$,

(iii) if $\left\{\left(x_{j}, \lambda_{j}\right)\right\}_{j=1}^{+\infty}$ is a sequence of $\partial Q \times[0,1]$ converging to $(x, \lambda)$ with $X=\lambda A x+$ $\lambda B^{n} x$ and $0 \leq \lambda<1$, then $\lambda_{j} A x_{j}+\lambda_{j} B^{n} x_{j} \in Q$ for $j$ sufficiently large,

(iv) $B$ is uniformly asymptotically regular with respect to $A$.

Then, $A+B$ has a fixed point in $Q$.

\section{Acknowledgements}

The authors would like to thank the referee for the insightful comments and suggestions. The first author would like to thanks The Thailand Research Fund for financial support and the second author is also supported by the Royal Golden Jubilee Program under Grant PHD/0282/2550, Thailand. Moreover, the second author the Thailand Research Fund for financial support under Grant BRG5280016.

\section{Authors' contributions}

The work presented here was carried out in collaboration between all authors. SP and AA defined the research theme. SP designed theorems and methods of proof and interpreted the results. AA proved the theorems, interpreted the results and wrote the paper. All authors have contributed to, seen and approved the manuscript. 


\section{Competing interests}

The authors declare that they have no competing interests.

Received: 30 January 2011 Accepted: 29 July 2011 Published: 29 July 2011

\section{References}

1. Agarwal, RP, O'Regan, D, Taoudi, MA: Browder-Krasnoselskii type fixed point theorem in Banach space. Fixed Point Theory Appl. (2010)

2. Agarwal, RP, Mehan, M, O'Regan, D: Fixed Point Theory and Applications. Cambridge University Press, Cambridge (2001)

3. Barroso, CS: Krasnoselskii's fixed point theorem for weakly continuous maps. Nonlinear Anal. 55, 25-31 (2003). doi:10.1016/S0362-546X(03)00208-6

4. Barroso, CS, Teixeira, EV: A topological and geometric approach to fixed point results for sum of operators and applications. Nonlinear Anal. 60, 625-650 (2005). doi:10.1016/.jna.2004.09.040

5. Ben Amar, A, Jeribi, A, Mnif, M: On a generalization of the Schauder and Krasnosel'skii fixed point theorems on Dunford-Pettis space and applications. Math Methods Appl Sci. 28, 1737-1756 (2005). doi:10.1002/mma.639

6. Burtun, TA: A fixed point theorem of Krasnoselskii. Appl Math Lett. 11, 85-88 (1998)

7. Burton, TA, Furumochi, T: Krasnoselskii's fixed point theorem and stability. Nonlinear Anal. 49, 445-454 (2002). doi:10.1016/50362-546X(01)00111-0

8. Fucik, S: Fixed point theorems for sum of nonlinear mappings. Commentationes Mathematicae Universitatis Carolinae. 9, 133-143 (1968)

9. Krasnoselskii, MA: Two remarks on the method of successive approximations. Uspekhi Math Nauk. 10, 123-127 (1955)

10. Krasnoselskii, MA: Topological Methods in the Theory of Nonlinear Integral Equations. Pergamon Press, New York (1964)

11. Krasnoselskii, MA, Zabrejko, PP, Pustyl'nik, Jl, Sobolevskii, Jl: Integral Operators in Spaces of Summable Functions. Nauka, Moscow (1966)

12. Latrach, K, Taoudi, MA, Zeghal, A: Some fixed point theorems of the Schauder and Krasnosel'skii type and application to nonlinear transport equations. J Diff Equ. 221, 256-271 (2006). doi:10.1016/j.jde.2005.04.010

13. O'Regan, D: Fixed point theory for the sum of two operators. Appl Math Lett. 9, 1-8 (1996)

14. O'Regan, D: Fixed point theory for weakly sequentially continuous mappings. Math Comput Model. 27, 1-14 (1998)

15. O'Regan, D: A fixed point theorems for condensing operators and applications to Hammerstein integral equations in Banach spaces. Comput Math Appl. 30, 39-49 (1995)

16. Park, S: Generalizations of the Krasnoselskii fixed point theorem. Nonlinear Anal. 67, 3401-3410 (2007). doi:10.1016/j. na.2006.10.024

17. Reinermann, J: Fixpunktsatze vom Krasnoselskii-typ. Math Z. 119, 339-344 (1971). doi:10.1007/BF01109885

18. Singh, SP: Fixed point theorems fora sum of nonlinear operators. Rend Accad Naz Lincei. 54, 1-4 (1973)

19. Taoudi, MA: Integrable solutions of a nonlinear functional integral equation on an unbounded interval. Nonlinear Anal. 71, 4131-4136 (2009). doi:10.1016/j.na.2009.02.072

20. Taoudi, MA: Krasnosel'skii type fixed point theorems under weak topology features. Nonlinear Anal. 72, 478-482 (2010). doi:10.1016/j.na.2009.06.086

21. Vijayaraju, P: Fixed point theorems for a sum of two mappings in locally convex spaces. Int J Math Math Sci. 17, 681-686 (1994). doi:10.1155/S0161171294000967

22. Goebel, K, Kirk, WA: A fixed point theorem for asymptotically nonexpansive mappings. Proc Am Math Soc. 35, 171-174 (1972). doi:10.1090/S0002-9939-1972-0298500-3

23. Cain, GL, Nashed, MZ: Fixed points and stability for a sum of two operators in locally convex spaces. Pacific J Math. 39, 581-592 (1971)

24. De Blasi, FS: On a property of the unit sphere in Banach spaces. Bull Math Soc Sci Math Roum. 21, 259-262 (1977)

25. Gu, F: Some convergence theorems of non-implicit iteration process with errors for a finite families of l-asymptotically nonexpansive mappings. Appl Math Comput. 216, 161-172 (2010). doi:10.1016/j.amc.2010.01.025

26. Chang, SS, Cho, YJ: The implicit iterative processes for asymptotically nonexpansive mappings. Nonlinear Funct Anal Appl. 1, 369-382 (2003)

27. Chidume, CE, Ofoedu, EU, Zegeye, H: Strong and weak convergence thorems for asymptotically nonexpansive mappings. J Mathods Anal Appl. 280, 364-374 (2003). doi:10.1016/50022-247X(03)00061-1

28. Gornicki, J: Weak convergence theorems for asymptotically nonexpansive mappings in uniformly convex Banach spaces. Comment Math Univ Carolin. 301, 249-252 (1989)

29. Tan, KK, Xu, HK: The nonlinear ergodic theorem for asymptotically nonexpansive mappings in Banach spaces. Proc Am Math Soc. 114, 399-404 (1992). doi:10.1090/S0002-9939-1992-1068133-2

30. Latrach, K, Taoudi, MA: Existence results for a generalized nonlinear Hammerstein equation on $L_{1}$ space. Nonlinear Anal. 66, 2325-2333 (2007). doi:10.1016/j.na.2006.03.022

31. Dunford, N, Schwartz, JT: Linear Operators, Part I: General Theory. Interscience Publishers, New York (1958)

32. O'Regan, D: Some fixed point theorems for concentrative mappings between locally convex linear topological spaces. Nonlinear Anal. 27, 1437-1446 (1996). doi:10.1016/0362-546X(95)00130-N

doi:10.1186/1029-242X-2011-28

Cite this article as: Arunchai and Plubtieng: On the Krasnoselskii-type fixed point theorems for the sum of continuous and asymptotically nonexpansive mappings in Banach spaces. Journal of Inequalities and Applications $20112011: 28$ 